\title{
LA PIRITA Y SU MANUFACTURA: ANÁLISIS DE CUATRO CONTEXTOS MESOAMERICANOS
}

\author{
Emiliano Melgar \\ Museo del Templo Mayor \\ Emiliano GallaGa \\ Centro INAH Chihuahua/The University of Arizona \\ Reyna Solís \\ Museo del Templo Mayor
}

\begin{abstract}
Resumen: En muchos sitios de Mesoamérica han sido recuperadas incrustaciones de pirita que dan forma a los espejos prehispánicos de mosaico. La mayoría de los estudios sobre estos objetos se han enfocado en su significado simbólico, morfología, comercio y uso, siendo escasos los trabajos que abordan las técnicas de manufactura y organización de la producción de dichos objetos. En este trabajo presentaremos los análisis tecnológicos que hemos aplicado a distintas incrustaciones de pirita de cuatro sitios mesoamericanos, apoyados en la arqueología experimental y en la aplicación del microscopio electrónico de barrido. De esta manera se han podido identificar con bastante precisión las herramientas empleadas en su elaboración que permitirán distinguir diferentes estilos tecnológicos.
\end{abstract}

PalabraS Clave: espejos de pirita, herramientas, talleres, Mesoamérica, arqueología experimental.

Summary: Several pyrite inlays that shaped Prehispanic mosaic mirrors have been recovered in many Mesoamerican sites. Recent researches on these objects have been focused on their symbolic meaning, morphology, trade and use, with few studies that address their manufacturing techniques and production organization. In this paper we present the technological analysis we applied to different pyrite inlays from four Mesoamerican sites, supported by experimental archaeology and technological instruments such as scanning electron microscope. In this way it has been possible to identify quite precisely the type of tools used in its manufacture, enabling us to distinguish different technological styles.

KEYWORDS: pyrite mirrors, tools, workshops, Mesoamerica, experimental archaeology.

RECEPCIÓN: 4 de octubre de 2013.

ACEPTACIÓN: 21 de enero de 2014. 



\title{
LA PIRITA Y SU MANUFACTURA: ANÁLISIS DE CUATRO CONTEXTOS MESOAMERICANOS
}

\author{
Emiliano Melgar \\ Museo del Templo Mayor \\ Emiliano Gallaga \\ Centro INAH Chihuahua/The University of Arizona \\ ReYna Solís \\ Museo del Templo Mayor
}

\section{Introducción}

Dentro del gran universo de objetos creados en el mundo prehispánico, uno de los que más han llamado la atención son los espejos o superficies reflejantes que se realizaban en materiales tan diversos como la obsidiana, la hematita y la pirita (Blainey, 2007; Gallaga, 2001; Pereira, 2008; Salinas, 1995). Debido a su estética, pero más aún por la destreza implicada en su manufactura, los investigadores concuerdan en que la cantidad de horas-hombre requeridas para la producción de un solo espejo de mosaico debió haber sido enorme y que, probablemente, fue uno de los objetos más complejos producidos por los artesanos prehispánicos (Blainey, 2007; Di Peso, 1974; Furst, 1966; Gallaga, 2001; Kidder et al., 1946; Pereira, 2008; Smith y Kidder, 1951; Woodward, 1941; Zamora, 2002a, 2002b). Por ello no es de extrañar que los espejos fueran considerados por Kirchhoff (1943) como uno de los criterios o elementos a considerar para definir a la región mesoamericana.

Si bien se reconoce la dificultad en la elaboración de estos espejos y la importancia que tenían como objetos de prestigio y mágico-religiosos, son muy pocas las investigaciones sobre sus técnicas de manufactura y organización de su producción.

En este trabajo, no nos avocaremos a los espejos per se, sino a los análisis tecnológicos que hemos aplicado a distintas incrustaciones de pirita de cuatro sitios mesoamericanos: Chiapa de Corzo y Tenam Puente en Chiapas, Teotihuacan en el Estado de México y Tenochtitlan en el Distrito Federal. El estudio lo realizamos apoyados en la arqueología experimental y la caracterización de huellas de manufactura con microscopía estereoscópica y con microscopía electrónica de barrido. De esta manera mostraremos cómo hemos podido identificar con bastante precisión las herramientas empleadas en su elaboración y distinguir diferentes tradiciones tecnológicas que reflejan las diferentes regiones, culturas y estilos de los cuales proceden las piezas. 


\section{Características de la pirita}

La pirita es un mineral compuesto de hierro y azufre perteneciente a los sulfuros de hierro $\left(\mathrm{Fe} \mathrm{S}_{2}\right.$ ). Tiene una coloración de amarillo metálico y una dureza de 6 a 6-5 en la escala de Mohs. La forma predominante de sus cristales es cúbica (Lagomarsino, 2008: 121). Debido a su inestabilidad, la acción del agua y el oxígeno pueden transformarla en otros minerales como el óxido de hierro (limonita y siderita), por lo cual difícilmente se conserva en buen estado en los contextos arqueológicos (Zamora, 2002a; 2002b: 695).

En cuanto a su origen, se han identificado siete yacimientos o minas prehispánicas en las que se infiere se extraía pirita junto con otros materiales. El primero de ellos son las minas de Chalchihuites en Zacatecas, además de la Sierra Gorda en la Huasteca, la cuenca del Río Balsas en Guerrero, las áreas de Huehuetenango, Aguacatán y Quetzaltenango en Guatemala; y por último, las Cockscomb Mountains en Belice, aunque seguramente no fueron los únicos yacimientos explotados (Kelley, 1971; Maldonado, 1980; Langenscheidt, 1988; Weigand, 1995; 1997; Kovacevich et al., 2002: 337; Zamora, 2002a; Gallaga, en prensa).

\section{Los espejos de pirita}

Dentro de los objetos de pirita creados por los artesanos prehispánicos, los espejos de mosaico son definitivamente los que más han acaparado la atención, un tanto por su estética, pero más aún por la destreza implicada en su manufactura. Los investigadores concuerdan en que la cantidad de horas-hombre requeridas para la producción de un solo espejo de pirita debió haber sido enorme y que, probablemente, fue uno de los objetos más complejos producidos por los artesanos prehispánicos (Woodward, 1941; Kidder et al., 1946; Smith y Kidder, 1951; Furst, 1966; Di Peso, 1974; Gallaga, 2001; Zamora, 2002a, 2002b; Blainey, 2007; Pereira, 2008).

En general, un espejo de pirita consiste de cuatro elementos básicos que describiremos a continuación (figura 1):

1) Base: Generalmente la base se hacía de piedra, sobre todo de areniscas, pizarras o esquistos, y sobre ella se montaban las placas de pirita. También se han reportado bases de cerámica y madera. Por lo general, las bases son redondas, pero se han registrado algunas rectangulares e, incluso, triangulares, aunque esta última forma es muy poco frecuente (Gladwin et al., 1965; Gallaga, 2001, en prensa; Zamora, 2002a, 2002b; Blainey, 2007; Pereira, 2008). Es posible que sus dimensiones variaran, dependiendo del uso, actividad y/o significado.

Las bases pueden medir entre 7 y $30 \mathrm{~cm}$ de diámetro y contar con un promedio de 8 a $10 \mathrm{~mm}$ de espesor (Gallaga, 2001, en prensa; Blainey, 2007). Los bordes de estas bases pueden ser biselados o perpendiculares a la superficie. Los bordes biselados pueden situarse al frente o en la parte posterior del espejo y, en algunas 


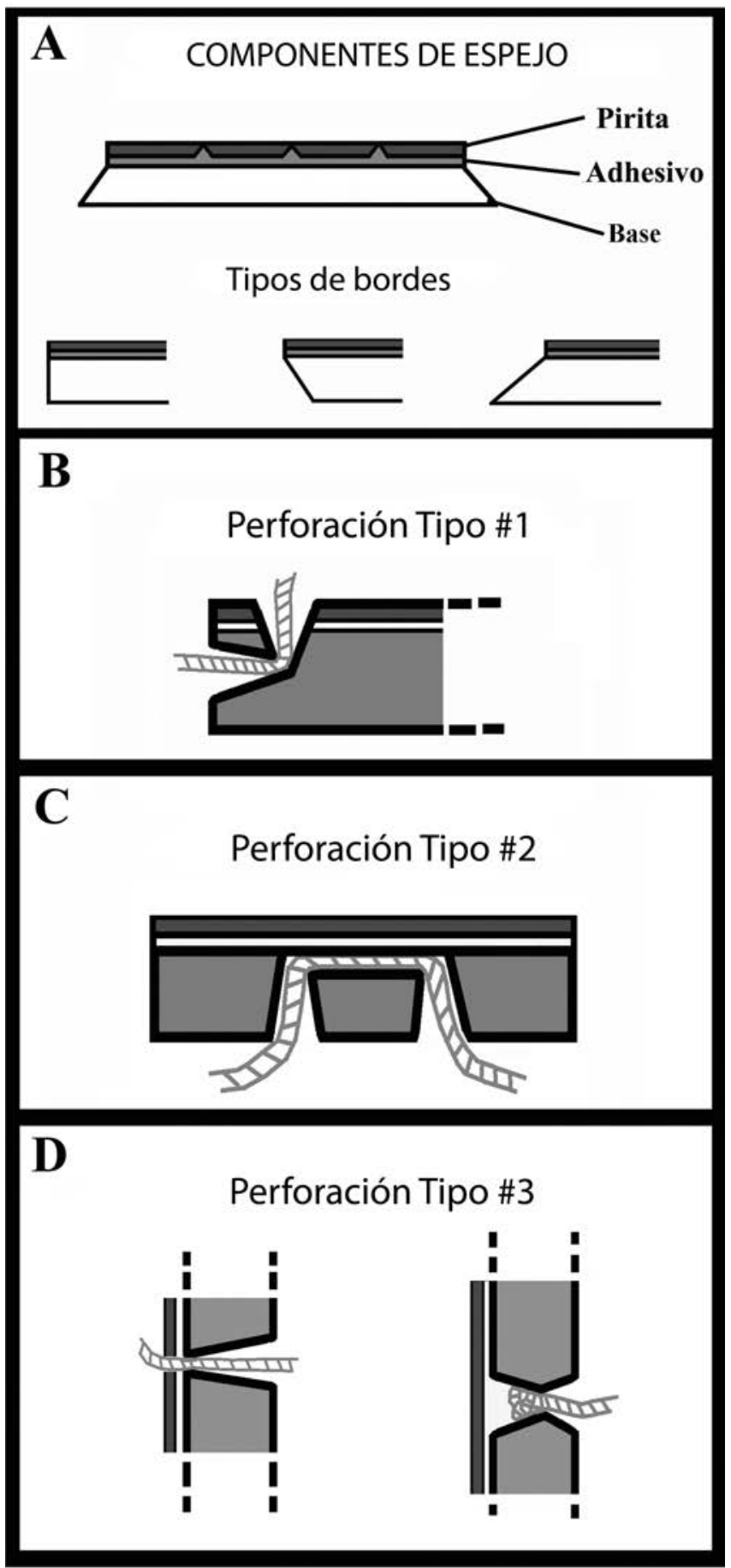

Figura 1. Componentes de un espejo de pirita y tipos de perforación (de Emiliano Gallaga). 
ocasiones, se localizan decorados con estuco pintado o con la técnica de pseudocloisonné. De igual forma, la porción posterior del espejo puede encontrarse decorada, ya sea con estuco pintado, pintada o tallada directamente (Ekholm, 1945; Kidder et al., 1946; Smith y Kidder, 1951; Di Peso, 1956, 1974; Gladwin et al., 1965; Furst, 1966; Kelley, 1971; Blainey, 2007).

2) Capa adhesiva: Existen al menos dos tipos de adhesivos. Uno de ellos está compuesto de un barro mezclado con un pegamento orgánico (Kidder et al., 1946: 126), el cual ha sido identificado en piezas de pirita del Lago Amatitlán mediante microscopía electrónica de barrido y mapeo de elementos químicos (Nelson et al., 2009: 3-5). El otro adhesivo está compuesto de una mezcla de brea que fue identificada durante los trabajos de conservación y restauración de un espejo de pirita con decoración de turquesa que fue localizado al centro del Palacio Quemado en el sitio de Tula, Hidalgo (Magar y Meehan, 1995, 2001: 7). En algunos espejos, la capa tiene un color amarillento que ha hecho suponer la utilización de algún adhesivo orgánico hecho a base de resina. Dependiendo del espejo y de las teselas de pirita, la capa adhesiva puede variar en su espesor de 1 a $3 \mathrm{~mm}$.

3) Teselas de pirita: Debido a sus características, la pirita es probablemente el material que consume más tiempo y que requiere de mayor habilidad en el proceso de producción. El número de teselas o placas usadas en cada espejo varía para cada objeto; hay desde una sola pieza (Furst, 1966), hasta 40 y 50 (Taube, 1992; Turner, 1992; Blainey, 2007). En términos generales, las dimensiones de las teselas pueden variar de 1 a $4 \mathrm{~cm}^{2}$, mientras que su espesor promedia entre los 2 y $4 \mathrm{~mm}$. Esta variabilidad puede ser a causa del tipo de materia prima, así como del tamaño y diseño del espejo.

4) Perforaciones: Otra característica de los espejos de pirita no menos importante son las perforaciones realizadas en él; con ello inferimos la forma de usarse o portarse (Gallaga, 2001, en prensa). En general, se describen dos tipos de perforaciones: las realizadas en los bordes y aquellas localizadas al centro del disco. El uso combinado de estos dos tipos de perforación es frecuente y suele utilizarse para incluir decoración o para combinar usos (Kidder et al., 1946; Smith y Kidder, 1951; Taube, 1992; Blainey, 2007; Pereira, 2008).

\section{Estudios previos sobre las técnicas de manufactura de los mosaicos de pirita}

Muchos estudios se han enfocado principalmente en el significado simbólico de los espejos de mosaico de pirita, su morfología, comercio y uso (Kidder et al., 1946; Pires-Ferreira, 1975; Pires-Ferreira y Evans, 1978; Taube, 1992; Sugiyama, 1992; Fialko, 2000; Gallaga, 2001; Zamora, 2002a, 2002b; Mata Amado, 2003; Blainey, 2007; Pereira, 2008), siendo escasos los trabajos que abordan las técnicas de manufactura empleadas en su elaboración. Por este desconocimiento de 
su producción, cuando se hallan mosaicos de pirita en los sitios arqueológicos generalmente se asume que fueron importados de otros lugares y que su elaboración y armado tan preciso debieron requerir una gran cantidad de tiempo de trabajo invertido (Smith y Kidder, 1951: 44).

A pesar de ello, existen algunos investigadores que proponen el uso de esmeril (óxido de aluminio), hematita, arena y ocre para desgastar y pulir los mosaicos de pirita, basados principalmente en las pocas referencias que hay de las herramientas empleadas por los artesanos lapidarios prehispánicos (Lunazzi, 1996: 3). Incluso hay quienes proponen el empleo de algún tipo de compás para lograr la forma perfectamente circular de las bases del espejo (Smith y Kidder, 1951: 44), hechas generalmente en arenisca. Sin embargo, estas propuestas son hipotéticas, pues carecen de experimentos y comparación de huellas de manufactura que las confirmen. Aún más escasos son los estudios de las evidencias de producción de mosaicos de pirita, destacando los realizados en Aguateca y en Cancuén: en el primer sitio los materiales proceden de los contextos residenciales de élite y administrativos y fueron clasificados en nueve nódulos de materia prima, 77 productos en proceso o reciclaje y 484 productos completos (455 piezas de mosaicos, 19 formas tridimensionales y 10 placas) (Zamora, 2002b: 696). A pesar de ello, solamente se señala que los materiales fueron redondeados en sus esquinas, cortados y pulidos por una o dos caras (Zamora, 2002b: 700), sin llegar a proponer con qué herramientas fueron trabajados.

Por otra parte, en Cancuén, fueron recuperadas en unidades domésticas de grupos no elitistas varias bases de espejo y herramientas de pedernal y cuarcita para alisar y pulir asociadas a piritas sin procesar, piezas semiterminadas y piezas pulidas para formar espejos (Kovacevich et al., 2002: 337; 2004: 885). A pesar de ello, poco se detalla sobre las herramientas empleadas en los espejos de pirita, ya que su interés se centra en la organización de la producción de las piezas, señalando que las primeras etapas del trabajo artesanal fueron llevadas a cabo por grupos domésticos no elitistas, mientras que las etapas finales las realizaban artesanos de la élite para monopolio y consumo de este grupo (Kovacevich et al., 2002: 343; 2004: 888; Kovacevich, 2007: 86-90).

\section{Análisis tecnológico de los objetos de pirita}

Debido a la escasez de información sobre la tecnología lapidaria en las fuentes históricas y a que las investigaciones sobre la elaboración de objetos de pirita generalmente se han basado en propuestas que no han pasado del nivel hipotético, con excepción de algunos intentos de carácter personal para reproducir alguna tesela (Ekholm, 1973; Gallaga, en prensa; Zamora, 2002a), consideramos que la arqueología experimental puede ayudar a resolver nuestro problema de estudio.

Según sus preceptos, en las sociedades humanas toda actividad se encuentra normada; por lo cual, los artefactos son usados o producidos de acuerdo con 
esquemas determinados que les proporcionan características específicas (Ascher, 1961: 807; Gándara, 1990: 51). Ello implica que la elaboración o utilización de objetos similares, siguiendo los mismos patrones, deben producir resultados idénticos (Ascher, 1961: 793; Coles, 1979: 171; Lewenstein, 1987: 7; Velázquez, 1999: 2). De esta manera y siguiendo el criterio uniformista, se postula que la utilización de una herramienta particular, hecha de un determinado material, empleada de una manera específica y bajo ciertas condiciones, dejará rasgos característicos y diferenciables entre sí (Binford, 1977: 7; Tringham, 1978: 180; Velázquez, 1999: 2; 2007: 23).

Con ello en mente y con el fin de resolver las interrogantes derivadas de la carencia de información sobre los métodos o técnicas prehispánicas empleadas en la producción de objetos lapidarios, en el 2004 se creó el Taller de Arqueología Experimental en Lapidaria con sede en el Museo del Templo Mayor dentro del proyecto "La lapidaria del Templo Mayor: estilos y tradiciones tecnológicas" (Melgar, 2006; 2009a; Melgar et al., 2010; Melgar y Solís, 2011). En dicho taller se han reproducido los diferentes tipos de modificaciones que presentan los objetos (tabla 1). Para ello hemos empleado herramientas y procesos referidos en las fuentes históricas (Durán, 1951; Sahagún, 1956), hallados en contextos arqueológicos (Feinman y Nicholas, 1993; Hohmann, 2002; Lewenstein, 1987; Moholy-Nagy, 1997; Pastrana, 1998) y propuestas de algunos investigadores (Athié, 2001; Charlton, 1993; Digby, 1964; Gazzola, 2007; Gómez, 2000; Mirambell, 1968; Semenov, 1957).

\begin{tabular}{|c|c|}
\hline MODIFICACIÓN & HERRAMIENTAS \\
\hline $\begin{array}{l}\text { Desgastes } \\
\text { de superficies }\end{array}$ & $\begin{array}{l}\text { Basalto, andesita, riolita, arenisca, caliza, granito y pizarra, } \\
\text { adicionando agua y ocasionalmente arena. }\end{array}$ \\
\hline Cortes & $\begin{array}{l}\text { Arena, agua y tiras de piel o cuerdas vegetales. } \\
\text { Herramientas líticas de pedernal y obsidiana. }\end{array}$ \\
\hline Perforaciones & $\begin{array}{l}\text { Abrasivos (arena, ceniza volcánica, polvo de obsidiana, polvo de } \\
\text { pedernal y polvo de cuarzo), animados con ramas de carrizo, } \\
\text { adicionando agua. } \\
\text { Herramientas líticas de pedernal y obsidiana. }\end{array}$ \\
\hline Calados & $\begin{array}{l}\text { Abrasivos (arena, ceniza volcánica, polvo de obsidiana, polvo de } \\
\text { pedernal y polvo de cuarzo), animados con ramas de carrizo de gran } \\
\text { diámetro, adicionando agua. }\end{array}$ \\
\hline Incisiones & Herramientas líticas de pedernal y obsidiana. \\
\hline Acabados & $\begin{array}{l}\text { Pulidos con abrasivos, agua y trozos de piel. } \\
\text { Bruñidos con trozos de piel en seco. } \\
\text { La aplicación de ambos acabados. }\end{array}$ \\
\hline
\end{tabular}

TABlA 1. Tipo de modificaciones y herramientas empleadas (por Emiliano Melgar). 
Las huellas resultantes son comparadas sistemáticamente con las de los objetos arqueológicos a simple vista, con ayuda de una lupa de 20x y a través del empleo de un microscopio estereoscópico a 10x, 30x y 63x, así como con microscopía electrónica de barrido (MEB) a 100x, 300x, 600x y 1000x, bajo parámetros constantes (modo HV, $10 \mathrm{~mm}$ de distancia, señal SEI, voltaje de $20 \mathrm{kV}$ y un haz de 47). Así, se describen las características superficiales observadas como la dirección y tamaño de bandas y líneas, su rugosidad y textura, siguiendo la misma metodología propuesta por Velázquez Castro (2007) para el estudio de los objetos de concha pero adecuada a objetos lapidarios. El microscopio óptico empleado está en el taller de arqueología experimental en concha del Museo del Templo Mayor y el microscopio electrónico de barrido se encuentra en el laboratorio de microscopía electrónica de la Subdirección de Laboratorios y Apoyo Académico del INAH, a cargo del ingeniero Gerardo Villa.

\section{Materiales analizados}

Los objetos estudiados corresponden a teselas de pirita de dos espejos que proceden de Chiapas. Uno de los espejos, de forma cuadrangular, fue recuperado en la Tumba 1 del Montículo 11 de Chiapa de Corzo, fechada para el Preclásico Medio (700-500 a.C.) (Bachand y Lowe, 2011); mientras que el segundo, de forma circular, proviene de la Tumba 10 del Edificio 21 de Tenam Puente, la cual está fechada para el Clásico Tardío (Martínez, 2010: 76-77). De cada uno fueron seleccionadas varias incrustaciones que tuvieran buen estado de conservación en sus superficies y bordes para que pudieran realizarse los análisis de las huellas de manufactura (figuras $2 \mathrm{a}$ y $2 \mathrm{~b}$ ).

También fueron analizadas tres incrustaciones circulares de pirita procedentes de Teopancazco (figura 2c), uno de los centros de barrio en Teotihuacan del periodo Clásico (200 a.C.-600 d.C.). El cuarto y último grupo de piezas incluido en este estudio fueron 35 incrustaciones semiesféricas del Templo Mayor de Tenochtitlan: siete forman parte del mosaico de la serpiente Xiuhcóatl que está hecha en pedernal y decorada con mosaicos de turquesa y pirita, encontrada en la Cámara III (figura 2d) y fechada para la Etapa IVa, que va de 1440 a 1469 d.C. Otras 26 forman las pupilas de trece máscaras cráneo de las Ofrendas 6, 11, 13, 17 y 20, y las dos restantes forman una de las pupilas de las esculturas de Tláloc en serpentina y de Xiuhtecuhtli en travertino (figuras 2e, $2 \mathrm{f}, 2 \mathrm{~g}$ y $2 \mathrm{~h}$ ) de la Cámara II. Estas últimas ofrendas están fechadas para la Etapa IVb, que va de 1469 a 1481 d.C.

\section{Resultados obtenidos}

Todas las incrustaciones de los dos espejos presentaron una superficie frontal alisada y lustrosa cruzada por algunas rayas rectas algo difusas (figuras 3a y 3e). 


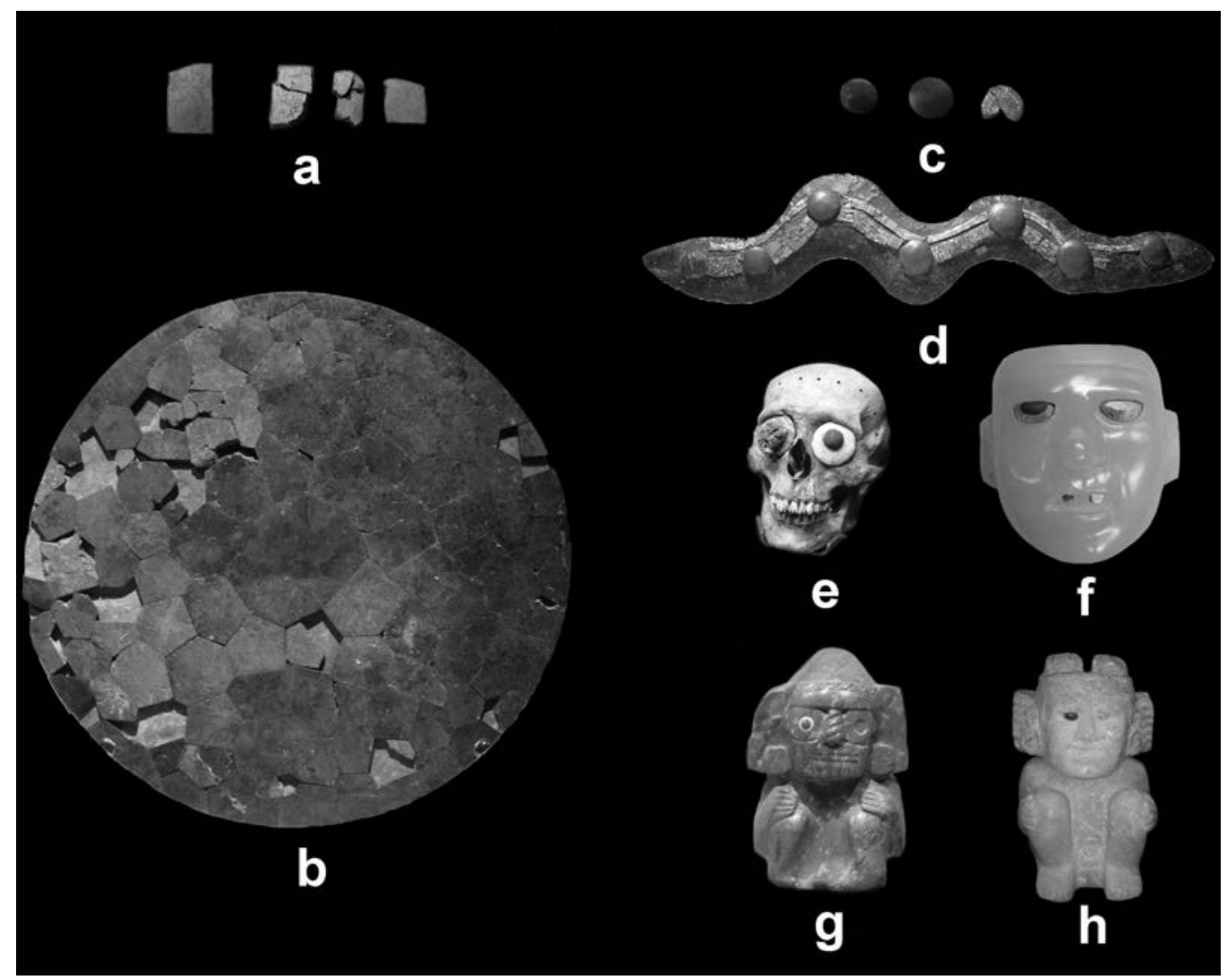

Figura 2. Materiales analizados: a) teselas de espejo de Chiapa de Corzo, b) espejo de Tenam Puente, $c$ ) incrustaciones circulares de Teopancazco, d) serpiente Xiuhcóatl, e) cráneos ofrenda, f) máscara de travertino, g) esculturas Tláloc de serpentina, y $h$ ) escultura de Xiuhtecuhtli (fotografía realizada por Emiliano Melgar).

En el caso de las piezas de Chiapa de Corzo, se observaron bandas rectas algo difusas que miden $33 \mu \mathrm{m}$ de anchura aproximadamente (figura $3 \mathrm{c}$ ), las cuales son similares a los desgastes experimentales hechos con lajas o metates de riolita y bruñidos con piel (figuras 3b y 3d). En contraste, las piezas de Tenam Puente mostraban amplias bandas aplanadas y difusas de $100 \mu \mathrm{m}$ de espesor sobre una textura rugosa (figura $3 \mathrm{~g}$ ), las cuales coinciden con los desgastes experimentales hechos con lajas o metates de basalto y con el empleo de arena como abrasivo, así como bruñido con piel (figuras $3 f$ y $3 \mathrm{~h}$ ). Cabe señalar que en ambos espejos las bandas de desgaste fueron más difusas en las caras frontales, debido al lustre producto de la aplicación de acabados, que en las dorsales, las cuales no fueron abrillantadas por estar en contacto con el soporte y el adhesivo. 

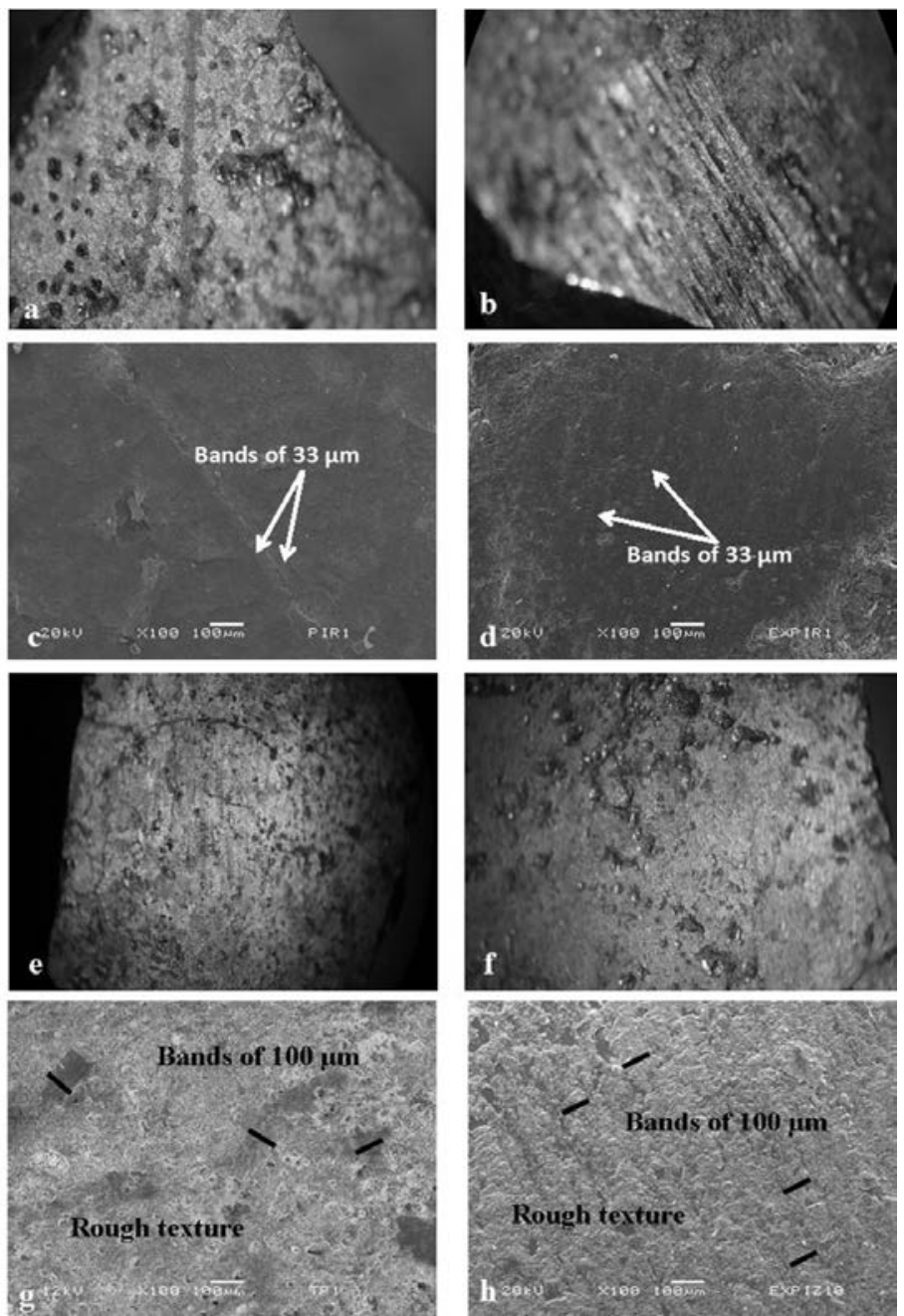

Figura 3. a) Superficie de tesela de Chiapa de Corzo, b) pulido experimental con riolita y bruñido con piel $(10 \mathrm{x}), c)$ superficie arqueológica (100x), d) superficie experimental (100x), e) superficie de tesela de Tenam Puente, $f$ ) pulido experimental con basalto y arena, y bruñido con piel (10x),

g) superficie arqueológica (100x), y h) superficie experimental (100x)

(fotografías realizadas por Emiliano Melgar y Gerardo Villa).

En cuanto a las incrustaciones circulares del barrio de Teopancazco, en Teotihuacan, las tres piezas presentan las superficies sumamente aplanadas y lustrosas (figura 4a), cruzadas por difusas bandas de $66 \mu \mathrm{m}$ de espesor (figura 4c), sobre las cuales hay finas bandas rectas-paralelas de entre 2 y $5 \mu \mathrm{m}$ de anchura. Estos rasgos son similares a los desgastes experimentales hechos con lajas o metates de andesita, pulidos con nódulos de pedernal y bruñidos con piel (figuras $4 \mathrm{~b}$ y $4 \mathrm{~d}$ ).

Respecto a las 35 incrustaciones semiesféricas de Tenochtitlan, en todos los casos las piezas presentan las superficies alisadas y lustrosas con varios rayones 
rectos-paralelos que se entrecruzan (figura 4e), los cuales miden $100 \mu \mathrm{m}$ de espesor (figura $4 \mathrm{~g}$ ). Estos rasgos se parecen a los desgastes experimentales hechos con basalto y bruñidos con piel (figuras $4 \mathrm{f}$ y $4 \mathrm{~h}$ ).
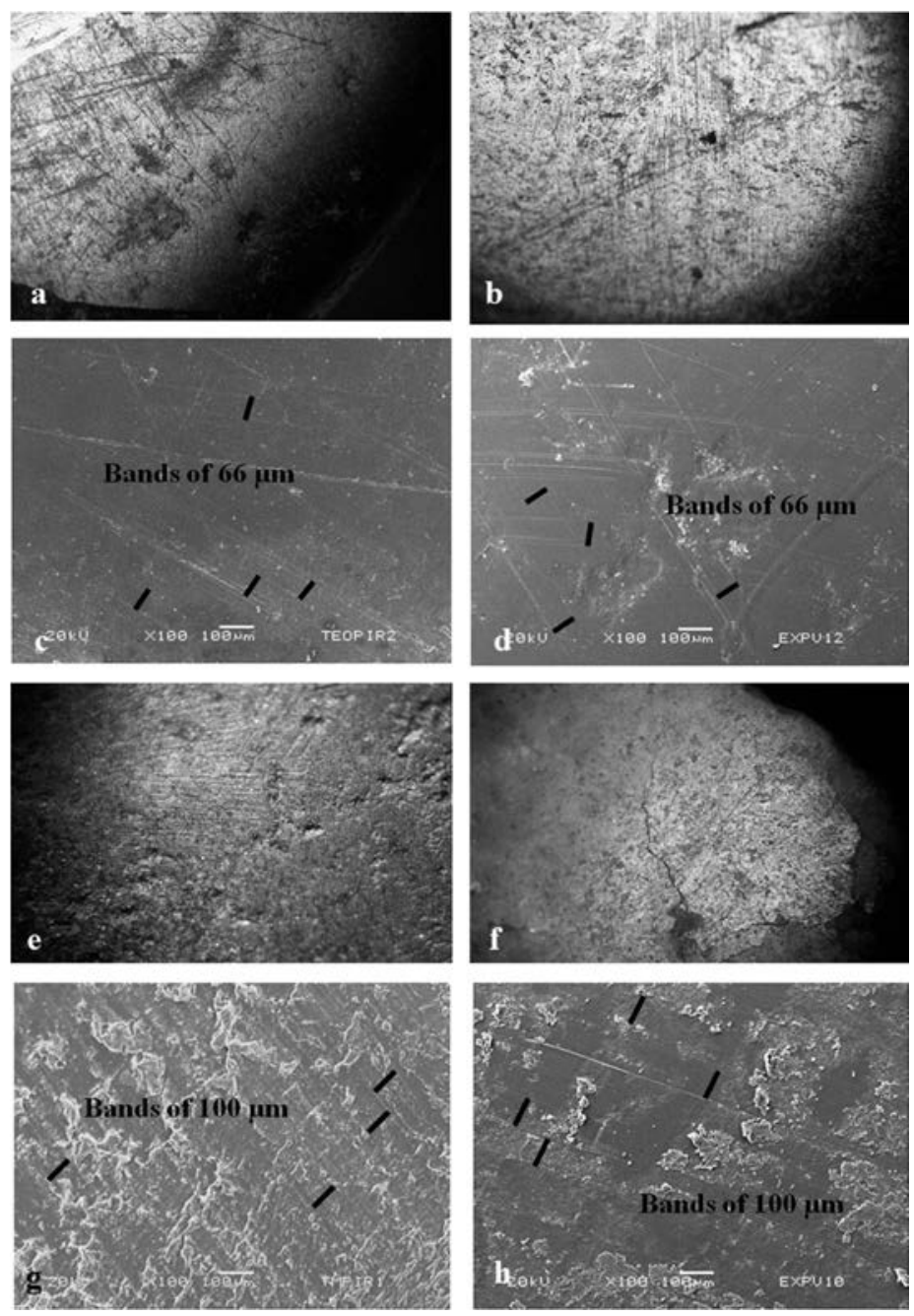

Figura 4. a) Superficie de tesela circular de Teopancazco, Teotihuacan, b) pulido experimental con andesita y bruñido con pedernal (10x), c) superficie arqueológica (100x),

d) superficie experimental (100x), e) superficie teselas de Templo Mayor, $f$ ) pulido experimental con basalto y bruñido con piel (10x), g) superficie arqueológica (100x), y h) superficie experimental (100x) (fotografías realizadas por Emiliano Melgar y Gerardo Villa).

En el caso de los bordes, todas las piezas de los dos espejos presentaron bordes rectos bastante rugosos (figuras 5a y 5b), cruzados por finas líneas de entre 
0.5 y $2 \mu \mathrm{m}$ de anchura (figuras $5 \mathrm{~d}$ y $5 \mathrm{e}$ ), similares a los obtenidos en los cortes experimentales hechos con lascas o navajillas de obsidiana (figura 5g). En contraste, todas las incrustaciones circulares tenían los bordes alisados y lustrosos (figura 5c), cruzados por bandas rectas-paralelas de 2 a $5 \mu \mathrm{m}$ de espesor (figura $5 f)$, los cuales coinciden con los cortes hechos con herramientas de pedernal (figura 5h). Mientras que las incrustaciones semiesféricas de Tenochtitlan no presentan bordes hechos por corte, ya que su silueta fue realizada por desgaste.
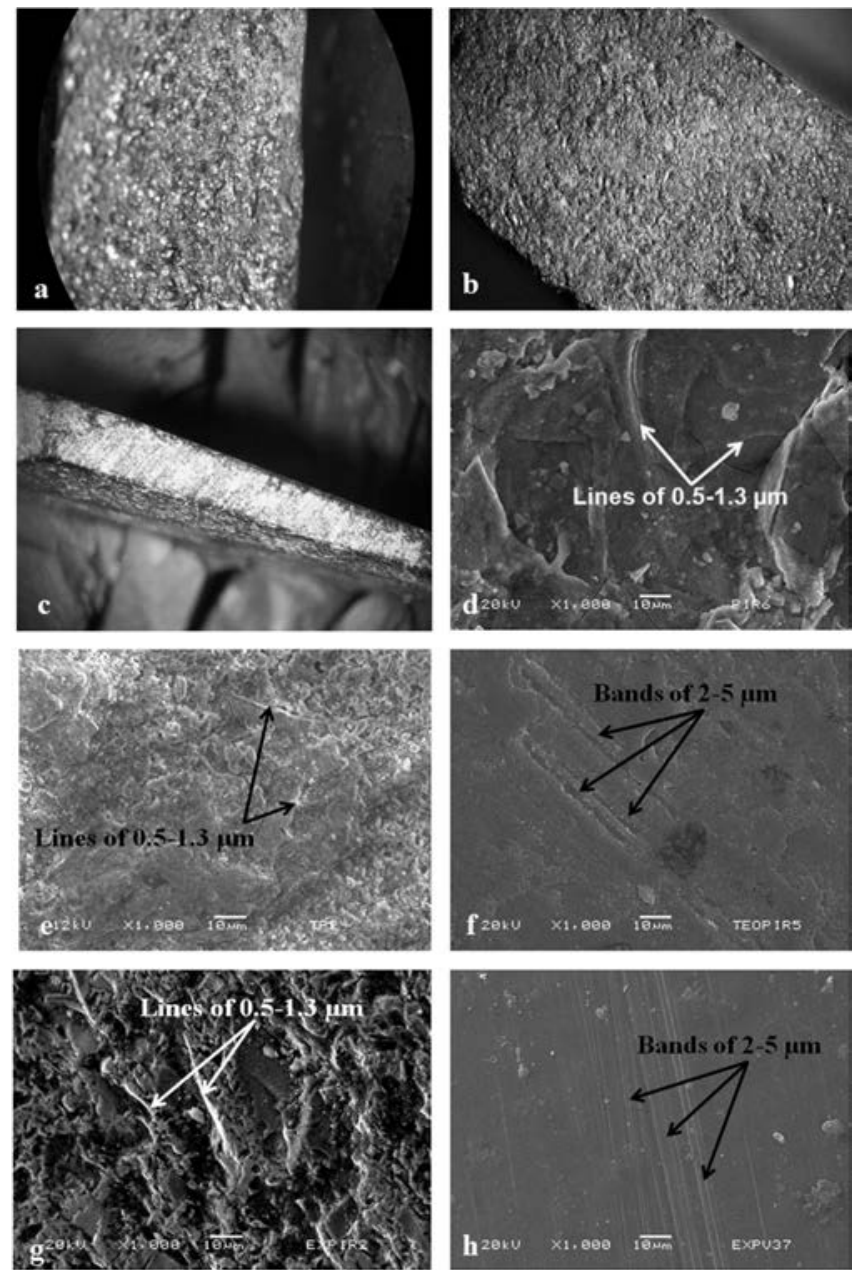

Figura 5. a) Borde de tesela de Chiapa de Corzo (10x), b) borde de tesela de Tenam Puente (10x),

c) borde de pieza de Teopancazco (10x), d) borde de Chiapa de Corzo (1000x), e) borde de Tenam Puente (1000x), $f$ ) borde de Teopancazco (1000x),

g) corte experimental con obsidiana (1000x), y h) corte experimental con pedernal (1000x) (fotografías realizadas por Emiliano Melgar y Gerardo Villa). 


\section{Discusión de resultados}

Como señalábamos al principio de este trabajo, la mayoría de las investigaciones sobre piezas de pirita, en especial los espejos de mosaicos, se han centrado en su aspecto simbólico, su variabilidad morfológica, su comercio y los contextos de uso o consumo de los mismos (Blainey, 2007; Fialko, 2000; Gallaga, 2001, en prensa; Kidder et al., 1946; Mata Amado, 2003; Pereira, 2008; Pires-Ferreira, 1975; Pires-Ferreira y Evans, 1978; Sugiyama, 1992; Taube, 1992; Zamora, 2002a, 2002b). Ello contrasta con los pocos estudios enfocados en sus técnicas de manufactura (Blainey, 2007: 177-179; Ekholm, 1973: 134; Gallaga, 2001, en prensa; Kovacevich et al., 2002: 343, 2004: 885-888; Lunazzi, 1996: 3; Zamora, 2002b: 696-700), los cuales en su mayoría retoman tal cual lo señalado en las fuentes históricas o especulan sobre las herramientas empleadas sin ofrecer argumentos sólidos.

El análisis tecnológico que presentamos nos ha permitido identificar con bastante precisión las herramientas empleadas en cada una de las modificaciones que presentan las piezas de pirita estudiadas. Con ello podemos corroborar o refutar las herramientas propuestas y reconstruir la secuencia de elaboración de cualquier pieza de pirita. De esta manera podemos recuperar información sobre su manufactura y demostrar que no solamente las evidencias directas de la producción (materias primas, piezas en proceso de trabajo, residuos y herramientas asociadas) halladas en contextos primarios (talleres) o secundarios (basureros y rellenos constructivos) son las únicas vías que nos permiten conocer a detalle los procesos de su elaboración. Como podemos apreciar, esta información la podemos recuperar de las mismas piezas terminadas, aun cuando procedan de contextos votivos o funerarios.

En cuanto a la organización de la producción de los objetos de pirita, los pocos estudios que abordan esta temática se basan en los parámetros de la especialización artesanal del modelo de Costin (1991): contexto (dependiente o independiente del control de la élite), concentración (centralización o dispersión de los grupos de trabajo), escala (tamaño de los grupos de trabajo) e intensidad (medio tiempo o tiempo completo). De esta manera señalan que la distribución dispersa de las evidencias de producción de pirita, en especial en unidades domésticas de grupos no elitistas, frente al hallazgo de piezas terminadas en contextos de élite, indica una especialización de medio tiempo llevada a cabo por artesanos que no eran miembros de la élite pero que producían para ella (Kovacevich et al., 2002: 343; Kovacevich, 2007: 86-90).

Cuando las evidencias de producción y las piezas terminadas se presentan exclusivamente en contextos residenciales elitistas y administrativos, se propone que su producción fue llevada a cabo por artesanos miembros de la élite porque las etapas finales de su manufactura requerían de conocimientos esotéricos y rituales especiales (Inomata, 2001: 321; 2007: 134-135; Kovacevich, et al., 2002: 344, 2004: 887-888; Kovacevich, 2007: 74-76 у 90). 
Como podemos apreciar en los estudios anteriores, la ubicación contextual y la distribución diferencial de las evidencias de producción (materia prima, piezas en proceso y piezas recicladas) de los mosaicos de pirita son los dos indicadores más importantes en que basan sus interpretaciones. Sin embargo, poco o nada nos dicen sobre el tamaño de los grupos de trabajo, mientras que la intensidad de la producción es inferida como de medio tiempo o de bajo volumen debido al hallazgo de materiales de otras actividades artesanales o domésticas como la elaboración de herramientas y la preparación de alimentos (Kovacevich et al., 2004: 887-888).

En el caso de los datos obtenidos en este análisis, podemos destacar que en cada sitio los objetos presentan una marcada estandarización morfológica y sobre todo tecnológica, que aunadas a lo exclusivo de los contextos (tumbas y ofrendas), permiten suponer una centralización de los grupos de trabajo bajo control de la élite. Asimismo, esta homogeneidad en formas y herramientas empleadas se ha postulado como indicador de que los grupos de trabajo debieron ser pequeños, concentrados y/o centralizados, ya que ello favorece el control y supervisión de la producción por parte de maestros artesanos y/o miembros de la élite (Costin, 1991: 16 y 40; Velázquez, 2007: 19-20).

Cabe señalar que para evaluar de manera relativa la intensidad o tiempo invertido de trabajo en la elaboración de un espejo de mosaico de pirita, el cual es considerado por muchos autores como muy alto o que requería una gran inversión de tiempo e insumos (Blainey, 2007; Di Peso, 1974; Furst, 1966; Gallaga, 2001, en prensa; Kidder et al., 1946; Pereira, 2008; Smith y Kidder, 1951; Woodward, 1941; Zamora, 2002a, 2002b), Emiliano Gallaga (en prensa) llevó a cabo una serie de experimentos utilizando arenisca para la base y pirita para el mosaico (figura 6). La elaboración de una base de piedra de arenisca de $9 \mathrm{~cm}$ de diámetro por $8 \mathrm{~mm}$ de espesor requirió de 87 horas de trabajo: tres horas de percusión con cantos rodados, 51 horas de desgaste con arena y agua sobre un metate de basalto, 8 horas de biselado usando los mismos instrumentos y 18 horas para hacer dos perforaciones al centro unidas por una acanaladura con ayuda de cristales de cuarzo montados en carrizos. La manufactura de una pieza de pirita de $1 \mathrm{~cm}^{2}$ por $4 \mathrm{~mm}$ de espesor requirió de 41 horas de trabajo por percusión con cantos rodados y desgaste con metate de basalto y arena.

Aunque son resultados preliminares, estos parámetros temporales o las horas/ hombre necesarias para la manufactura de un espejo de pirita de aproximadamente $9 \mathrm{~cm}$ de diámetro y un mosaico de 20 y 30 teselas de pirita, requieren de 900 a 1300 horas, o de 110 a 160 días de trabajo realizado por una sola persona. Por la cantidad de tiempo invertido, la accesibilidad de la materia prima y la habilidad requerida para su manufactura, podemos inferir que el o los artesanos debieron haber sido especialistas de tiempo completo.

Asimismo, llama la atención que las herramientas empleadas no siempre fueron las más eficientes, ya que el uso de abrasivos abate los tiempos de trabajo. Sin embargo, solamente las piezas de Tenam Puente presentaron los desgastes 


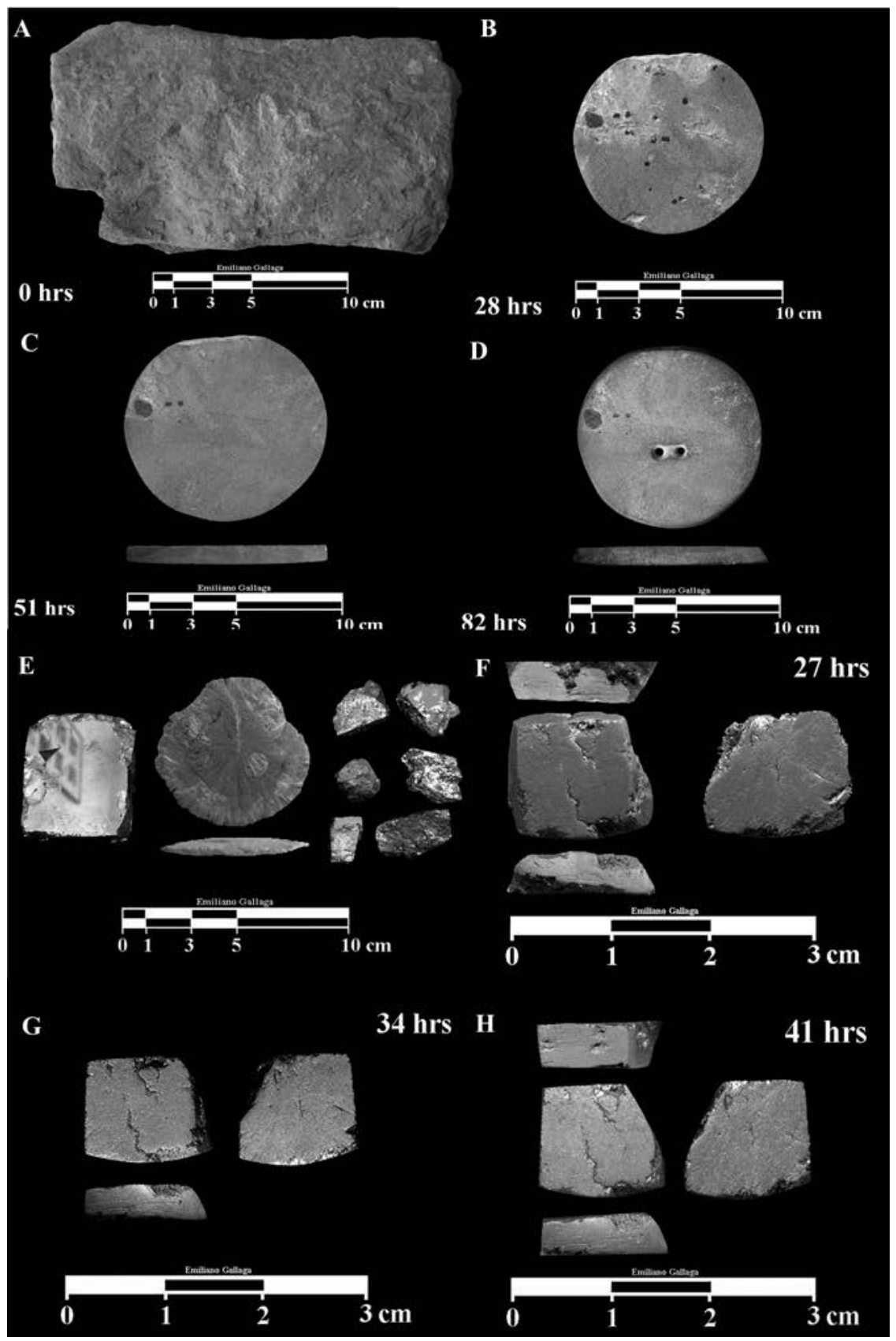

Figura 6. Proceso de manufactura experimental de espejo de pirita (fotografías realizadas por Emiliano Gallaga). 
con arena sobre basalto, mientras que en los demás sitios utilizaron solamente lajas o metates de basalto, andesita y riolita sin ayuda de algún tipo de abrasivo. Esto nos indica que los artesanos que elaboraban estos objetos no buscaban producir la mayor cantidad de piezas en el menor tiempo posible, ya que parte del valor de éstas se debe al tiempo de trabajo invertido en ellas (Melgar, 2009 b: 261-263; Widmer, 2009: 182). Esto no es de extrañar, pues se trata de bienes suntuarios o de prestigio, en los cuales no se escatiman tiempos ni insumos (Shimada, 1994: 25; Velázquez, 2007: 18). Además, la selección de determinadas herramientas para su elaboración no siempre depende de la cercanía geográfica o acceso a los materiales, muchas veces son establecidas por la tradición, la ideología, la economía política, la religión o la cultura (Gosselain, 1992: 580; Lemmonier, 1986: 153, 2002: 4; Melgar, 2009b: 5; Pfaffenberger, 1988: 249; Schiffer, 1992: 51; Velázquez, 2007: 22;).

En este sentido, llama la atención la variabilidad en las herramientas empleadas entre cada sitio. Estas diferencias tecnológicas quizás nos remiten a distintas tradiciones de manufactura o estilos tecnológicos regionales o locales propios de los zoques, teotihuacanos, mayas y aztecas. Esta idea se apoya en el supuesto de que cada cultura o grupo social tiene formas particulares y características de elaborar sus objetos, donde el estilo puede considerarse como la elección y repetición sistemática y normada de una determinada manera de hacer las cosas - por ejemplo, la producción de espejos de pirita—, a expensas de otras, en un espacio y tiempo determinados (Carr, 1995: 166; Velázquez, 2007: 22). De esta manera, el introducir la variable tecnológica al estilo nos permite profundizar en las formas en que una cultura se expresa a través de manufacturar piezas y qué tanto a dichos materiales les imprime su sello de localidad o identidad (Gosselain, 1992: 583; Wobst, 1977: 321).

Con ello en mente, sería interesante comparar las huellas de manufactura y herramientas empleadas en otros sitios y objetos lapidarios de las regiones estudiadas para tratar de caracterizar temporal y espacialmente estas tradiciones tecnológicas. Al respecto, podemos señalar que en el caso de las piezas de Teotihuacan y de Tenochtitlan, las herramientas identificadas las presentan otros objetos lapidarios de ambos sitios (Melgar 2006, 2009a; Melgar y Filloy, 2008; Melgar y Solís, 2011), muchos de ellos clasificados como de "estilo teotihuacano" (pendientes trapezoidales y figurillas antropomorfas) o de "manufactura mexica" (pectorales circulares Anáhuatl, cetros cilíndricos con remate globular de Techalotl, narigueras rectangulares de extremos bífidos de Xipe Totec, figurillas antropomorfas de Tláloc y mosaicos con las representaciones de Huitzilopochtli, Mixcóatl y Tlahuizcalpantecuhtli). Por ello, las tecnologías lapidarias de estos dos sitios podrían considerarse como propias de los teotihuacanos y de los mexicas respectivamente, mientras que de Chiapa de Corzo y Tenam Puente todavía haría falta realizar más estudios para poder llegar a estos mismos resultados. 


\section{Conclusiones}

Como hemos podido apreciar, los estudios tecnológicos de objetos lapidarios, apoyados en la arqueología experimental y la caracterización de huellas de manufactura con microscopía estereoscópica y microscopía electrónica de barrido, permiten conocer con precisión las herramientas empleadas en cada una de las modificaciones que presentan las piezas.

En este trabajo fueron analizadas las incrustaciones de pirita de cuatro sitios mesoamericanos: Chiapa de Corzo, Tenam Puente, Teotihuacan y Tenochtitlan. Los resultados obtenidos permitieron identificar que en cada sitio se trabajaban estas piezas con distintas herramientas, pero que su elaboración estaba estandarizada morfológica y tecnológicamente. Ello nos permite inferir algunos aspectos sobre la organización de su producción como el tamaño de los grupos de trabajo, los cuales debieron ser pequeños, concentrados y/o centralizados, lo cual favoreció el control y supervisión de su producción por parte de maestros artesanos y/o miembros de la élite. Los patrones de manufactura identificados son diferentes entre los sitios, donde en el caso de Teotihuacan y Tenochtitlan ha podido confirmarse que esta misma tecnología la presentan otros objetos lapidarios clasificados como de "estilo teotihuacano" o de "manufactura mexica", por lo cual podríamos considerarlos como propios de ambos sitios.

También podemos suponer que el tiempo de trabajo en la elaboración de los espejos de mosaico debió ser muy alto, ya que la manufactura de la base de arenisca y de una sola incrustación llevó más de 100 horas efectivas de trabajo, por lo cual, si consideramos que un mosaico tiene entre 20 y 30 teselas de pirita, se requerirían de 900 a 1300 horas, o de 110 a 160 días de trabajo de una sola persona.

Asimismo, cabe destacar que las herramientas identificadas no siempre fueron las más eficientes en términos de tiempo de trabajo, por lo cual este mayor trabajo invertido debió otorgarles mayor valor y estima, pues no debemos olvidar que se trata de objetos suntuarios o bienes de prestigio, en los cuales no se escatiman ni tiempos ni insumos. En particular resultó interesante establecer que los lapidarios prehispánicos están usando lascas de obsidiana y pedernal para realizar los cortes, en este caso de las piritas, método que incrementa en mucho los tiempos de producción de cualquier objeto que use este material (figura 7). Falta mucho por analizar, investigar y experimentar, para establecer otros métodos de corte o qué tan común fue la utilización de lascas para este efecto, pero podemos establecer que esta metodología de trabajo es bastante efectiva para determinar las cadenas de operación, las herramientas, y los tiempos en cuanto a manufactura de objetos prehispánicos.

Otro punto muy importante a destacar en las preferencias culturales de cada grupo artesanal nos obliga a cuestionar la selección de los instrumentos de trabajo basada en la dureza de los materiales, tanto de los objetos como de las herramientas. No debemos confundir la capacidad de un material "suave" para trabajar 


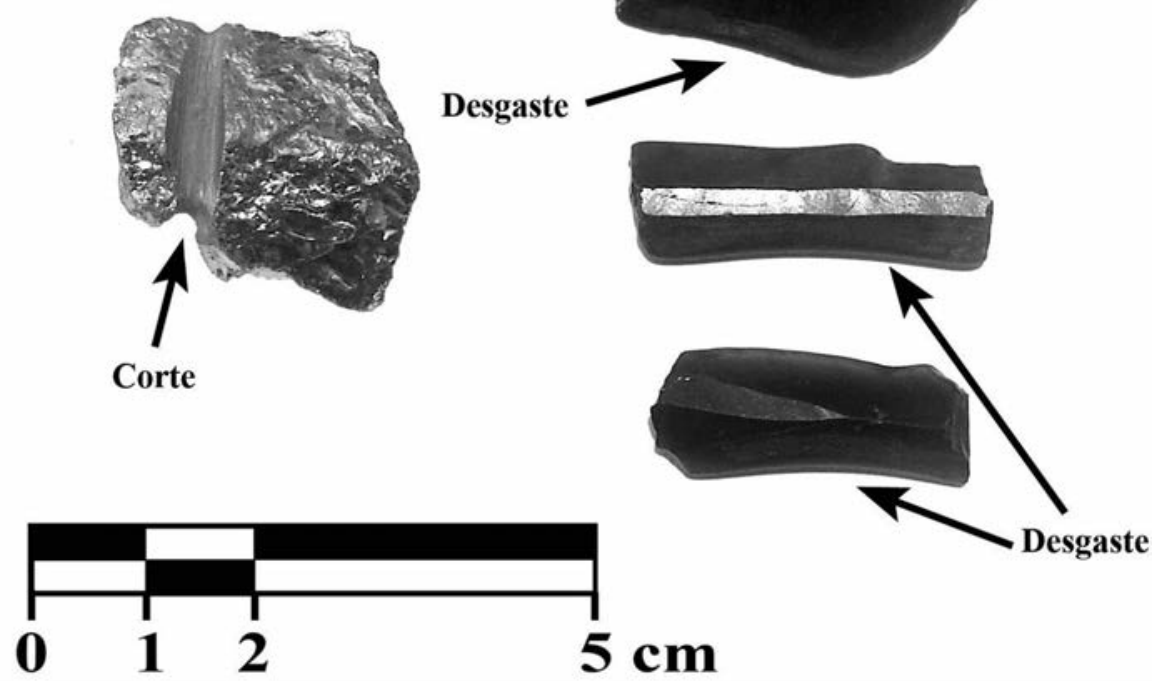

Figura 7. Corte experimental de pirita con lascas de obsidiana (fotografía realizada por Emiliano Gallaga).

materiales "más duros" con la medición de la dureza de una materia prima con la escala de Mohs, que solamente mide la capacidad de penetración de su superficie (posibilidad de rayarla con "una sola pasada") con determinados materiales de referencia. Si uno emplea decenas de lascas de obsidiana (con una dureza de 5 a 6 en la escala de Mohs) para cortar pirita (con 6 a 6.5 en la escala de Mohs), después de unas horas de trabajo es posible seccionar dicho material, como lo pudimos lograr en el taller de arqueología experimental en lapidaria. Lo anterior quedó confirmado al analizar las huellas de manufactura y encontrar trazos de este material en los bordes de las incrustaciones de los espejos de Chiapa de Corzo y de Tenam Puente. En otras palabras, no debemos descartar a priori el empleo de determinados instrumentos de trabajo solamente basados en la medida de su dureza con nuestros criterios occidentales. Al respecto, las evidencias arqueológicas en los talleres de jadeíta del área maya, en el Motagua, Cancuén, Calakmul y Copán demuestran que las lascas y navajas de obsidiana fueron los materiales preferidos para trabajar estas piedras verdes (Domínguez y Folan, 1999: 634-643; Kovacevich, 2007: 80-84; Rochette, 2009: 214-217), que en la escala de Mohs son más duras que la obsidiana. Nuevamente, quienes descartan el uso de ciertos materiales "suaves" por su dureza subestiman la capacidad de los lapidarios prehispánicos y 
contradicen los datos que nos proporcionan los talleres hallados en excavaciones arqueológicas. Por lo tanto, esperamos que, con los resultados obtenidos y apoyados en estos argumentos, se abran nuevas perspectivas de investigación al analizar la tecnología empleada en la elaboración de objetos lapidarios.

Para finalizar, esperamos que este tipo de estudios detallados y sistemáticos de las huellas de manufactura de objetos lapidarios se realicen en más colecciones y sitios de Mesoamérica, para delimitar con mayor precisión las distintas tradiciones tecnológicas que se desarrollaron en la época prehispánica. Solamente de esta manera podremos pasar de las propuestas hipotéticas del empleo de determinados instrumentos de trabajo, como el picoteo en obsidiana (Athié, 2001: 126-130) y el corte con fibras vegetales (de origen americano) en piedras verdes (Gazzola, 2007: 60-62), las cuales carecen de experimentos de contrastación entre las huellas y las herramientas planteadas que sustenten dichas aseveraciones.

\section{Agradecimientos}

Este trabajo no hubiera sido posible sin el apoyo y colaboración de Adrián Velázquez, del Museo del Templo Mayor, de Gerardo Villa, del Laboratorio de Microscopía Electrónica del INAH, y de los integrantes del taller de arqueología experimental en lapidaria (Mauricio Valencia, Isaac Ramírez, Mijaely Castañón, Hervé Monterrosa, Edgar Pineda, Viridiana Guzmán, Adriana Soto, Rebeca Mendoza y Tony Agis). Agradecemos también el apoyo del "Proyecto Arqueológico Chiapa de Corzo", dirigido por Bruce Bachand, Lynneth Lowe y Emiliano Gallaga, al "Proyecto Arqueológico Tenam Puente", dirigido por Gabriel Lalo Jacinto, y al proyecto "Teotihuacan: élite y gobierno", dirigido por Linda Manzanilla, del Instituto de Investigaciones Antropológicas de la UNAM.

\section{Bibliografía}

Ascher, Robert

1961 "Experimental Archaeology", American Anthropologist, vol. 63 (4): 793-816. Arlington, Virginia: American Anthropological Association.

Athie Islas, Ivonne

2001 "La obsidiana del Templo Mayor de Tenochtitlan", tesis de Licenciatura en Arqueología. México: Escuela Nacional de Antropología e Historia.

Bachand, Bruce y Lynneth Lowe

2011 "Chiapa de Corzo y los olmecas", Arqueología Mexicana, vol. XVIII (107): 7483. México: Raíces.

Blainey, Marc G.

2007 "Surfaces and Beyond: The Political, Economic, and Ideological Significance of Ancient Maya Iron Ore Mirrors", tesis de Maestría. Peterborough, Ontario, Canadá: Universidad de Trent. 
Binford, Lewis R.

1977 "General Introduction", For Theory Building in Archaeology. Essays on Faunal Remains, Aquatic Resources, Spatial Analysis, and Systemic Modeling, Lewis R. Binford (ed.). Albuquerque: Academic Press, pp. 1-10.

Carr, Christopher

1995 "Building a Unified Middle-Range Theory of Artifact Design", Style, Society and Person, C. Carr y J. E. Neitzel (eds.). Nueva York / Londres: Plenum Press, pp. 151-170.

Charlton, Cynthia Otis

1993 "Obsidian as Jewelry: Lapidary Production in Aztec Otumba, México", Ancient Mesoamerica, 4: 231-243. Cambridge: Cambridge University Press.

Coles, John

1979 Experimental archaeology. Londres: Academic Press.

Costin, Cathy Lynne

1991 "Craft Specialization: Issues in Defining, Documenting, and Explaining the Organization of Production", Archaeological Method and Theory, volume 3, Michael B. Schiffer (ed.). Tucson: The University of Arizona Press, pp. 1-56.

Digby, Adrian

1964 Maya Jades. Londres: The Trustees of the British Museum.

Di Peso, Charles

1956 The Upper Pima of San Cayetana del Tumacacari: An Archaeological Reconstruction of the Ootal of Pimeria Alta. Arizona: Dragoon, AZ (Amerind Foundation Publication, 7).

1974 Casas Grandes: A Fallen Trading Center of the Grand Chichimeca, vol. 1. Flagstaff: Amerind Foundation.

Domínguez Carrasco, María del Rosario y William J. Folan

1999 "Hilado, confección y lapidación: los quehaceres cotidianos de los artesanos de Calakmul, Campeche, México”, XII Simposio de Investigaciones Arqueológicas en Guatemala, 1998, Juan Pedro Laporte y Héctor L. Escobedo (eds.). Guatemala: Museo Nacional de Arqueología y Etnología, pp. 628-646.

Durán, Fray Diego

1951 Historia de las Indias de Nueva España e islas de Tierra Firme. México: Editorial Nacional, 2 tomos.

Ekholm, Gordon F.

1945 "A Pyrite Mirror from Queretaro, México", Carnegie Institution of Washington Notes for Middle American Archaeology and Ethnology, 53 (2): 178-181. Washington: Carnegie Institution.

1973 "The Archaeological Significance of Mirrors in the New World", Atti del XL Congresso Internazionale degli Americanisti, Roma-Genova, 1972, vol. 1: Abs- 
tractos de la $40^{\circ}$ Congreso Internacional de Americanistas. Rome-Genoa, Genova: Casa Editrice Tilgher, pp. 133-135.

Feinman, Gary M. y Linda M. Nicholas

1993 "Shell-Ornament Production in Ejutla. Implications for Highland-Coastal interaction in Ancient Oaxaca", Ancient Mesoamerica, 4: 103-119. Cambridge: Cambridge University Press.

Fialko, Vilma

2000 "El espejo del Entierro 49; morfología y texto jeroglífico", El sitio maya de Topoxté. Investigaciones en una isla del lago Yaxhá, Petén, Guatemala, Wolfgang W. Wurster (ed.). Mainz am Rhein, Germany: Verlag, pp. 144-149.

Furst, Peter T.

1966 "Shaft Tombs, Shell Trumpets and Shamanism: A Culture-Historical Approach to Problems in West Mexican Archaeology", tesis doctoral. Los Ángeles: Universidad de California.

Gallaga M., Emiliano

2001 "Descripción y análisis de los espejos de pirita del sitio de Snaketown, AZ, EU y su relación con Mesoamérica”, ponencia presentada en la XXVI Mesa Redonda de Antropología en la Ciudad de Zacatecas, Zacatecas, México.

En prensa "How to Make a Pyrite Mirror: An Application of Experimental Archaeology", Reflections of the Soul: Mirrors in the Mesoamerican Realm, Emiliano Gallaga y Marc Blainey (eds). Colorado: Colorado Press, pp. 29-60.

Gándara Vázquez, Manuel

1990 "La analogía etnográfica como heurística: lógica muestral, dominio etnográfico e historicidad”, Etnoarqueología. Primer Coloquio Bosch-Gimpera, Yoko Sugiura y Mari Carmen Serra Puche (eds.). México: Universidad Nacional Autónoma de México, Instituto de Investigaciones Antropológicas, pp. 43-82.

Gazzola, Julie

2007 "La producción de cuentas en piedras verdes en los talleres lapidarios de La Ventilla, Teotihuacan”, Arqueología, 36: 52-70. México: Raíces.

Gladwin, H. S., E. W. Haury, E. B. Sayles y N. Gladwin

1965 Excavation at Snaketown: Material Culture, $2^{\mathrm{a}}$ ed. Tucson: University of Arizona Press.

Gómez Chávez, Sergio

2000 "La Ventilla: un barrio de la antigua ciudad de Teotihuacan: Exploraciones y resultados”, tesis de Licenciatura en Arqueología. México: Escuela Nacional de Antropología e Historia.

Gosselain, Olivier P.

1992 “Technology and Style: Potters and Pottery among Bafia of Cameroon”, Man 27 (3): 559-583. Gran Bretaña: Royal Anthropological Institute of Great Britain and Ireland. 
Hohmann, Bobbi M.

2002 "Preclassic Maya Shell Ornament Production in the Belize Valley, Belize", tesis doctoral. Albuquerque: The University of New Mexico.

Inomata, Takeshi

2001 "The Power and Ideology of Artistic Creation. Elite Craft Specialists in Classic Maya Society”, Current Anthropology, 42 (3): 321-333. Chicago: University of Chicago Press (The Wenner-Gren Foundation for Anthropological Research).

2007 "Classic Maya Elite Competition, Collaboration, and Performance in Multicraft Production", Craft Production in Complex Societies. Multicraft and Producer Perspectives, Izumi Shimada (ed.). Utah: University of Utah Press, (Foundations of Archaeological Inquiry), pp. 120-133.

Kelley, J. Charles

1971 "Archaeology of the Northern Frontier: Zacatecas and Durango", Handbook of Middle American Indians: Archaeology of Northern Mesoamerica, Part two, vol. 11, Robert Wauchope (ed.). Austin: University of Texas Press, pp. 768801.

Kidder, Alfred V., Jesse D. Jennings y Edwin M. Shook

1946 Excavation at Kaminaljuyu, Guatemala. Washington: Carnegie Institution of Washington (Publicación, 561).

Kirchhoff, Paul

1943 "Mesoamérica; sus límites geográficos, composición étnica y caracteres culturales", Acta Americana, 1: 92-107. Washington: Sociedad Interamericana de Antropología e Historia.

Kovacevich, Brigitte

2007 "Ritual, Crafting, and Agency at the Classic Maya Kingdom of Cancuen", Mesoamerican Ritual Economy. Archaeological and Ethnological Perspectives, E. Christian Wells y Karla L. Davis Salazar (eds.). Boulder: University Press of Colorado, pp. 67-114.

Kovacevich, Brigitte, Tomás Barrientos, Michael Callaghan y Karen Pereira

2002 "La economía en el Reino Clásico de Cancuen: Evidencia de producción, especialización e intercambio", XV Simposio de Investigaciones Arqueológicas en Guatemala, 2001, J. P. Laporte, H. Escobedo y B. Arroyo (eds.). Guatemala: Museo Nacional de Arqueología y Etnología, pp. 333-349.

Kovacevich, Brigitte, Duncan Cook y Timothy Beach

2004 "Áreas de actividad doméstica en Cancuen: Perspectivas basadas en datos líticos y geo-químicos”, XVII Simposio de Investigaciones Arqueológicas en Guatemala, 2003, J. P. Laporte, B. Arroyo, H. Escobedo y H. Mejía (eds.). Guatemala: Museo Nacional de Arqueología y Etnología, pp. 876-891.

Lagomarsino, James

2008 A Pocket Guide to Rocks \& Minerals. Bath: Parragon. 
Langenscheidt, Adolphus

1988 Historia mínima de la minería en la Sierra Gorda. Windsor, Ontario, Canadá: Rolston-Bain, Windsor.

2006 "Los abrasivos en Mesoamérica”, Arqueología Mexicana, 14 (80): 55-60. México: Raíces.

2007 "Lapidaria mesoamericana, una reflexión sobre los abrasivos posiblemente usados para trabajar los chalchihuites duros", Arqueología, 36: 179-206. México: Raíces.

Lemonnier, Pierre

1986 "The Study of Material Culture Today: Toward and Anthropology of Technical Systems", Journal of Anthopological Archaeology, 5: 147-186. USA: Elsevier.

Lewenstein, Suzanne M.

1987 Stone Tools at Cerros. The Ethnoarchaeological and Use-wear Evidence. Austin: University of Texas Press.

Lunazzi, José J.

1996 "On the Quality of Olmec Mirrors and its Utilization", Proceedings of SPIE, Volume 2730. Second Iberoamerican Meeting on Optics, Guanajuato, Mexico, September 18-22, 1995, Daniel Malacara Hernández, Sonia E. Acosta Ortiz, Ramón Rodríguez Vera, Zacarías Malacara y Arquímedes A. Morales (eds.). Bellingham, Washington: SPIE Press, pp. 2-7.

Magar, Valerie y Patricia Meehan

1995 "Investigación para la interpretación y la conservación de un disco de mosaico de turquesa”, tesis de Licenciatura en Restauración de Bienes Muebles. México: Escuela Nacional de Conservación, Restauración y Museografía.

2001 "Consideration on the Conservation and Investigation of the Turquoise Mosaic Disk from the Palacio Quemado, Tula, México", ponencia presentada en la 66 Reunión Anual de la SAA, New Orleans.

Maldonado Cárdenas, Rubén

1980 Ofrendas asociadas a entierros del Infiernillo en el Balsas. México: Instituto Nacional de Antropología e Historia.

Martínez del Campo Lanz, Sofía

2010 Rostros de la divinidad. Los mosaicos mayas de piedra verde. México: Instituto Nacional de Antropología e Historia.

Mata Amado, Guillermo

2003 "Espejo de pirita y pizarra de Amatitlán”, XVI Simposio de Investigaciones Arqueológicas en Guatemala, 2002, J. P. Laporte, B. Arroyo, H. Escobedo y H. Mejía (eds.). Guatemala: Museo Nacional de Arqueología y Etnología, pp. 831-839.

Melgar Tísoc, Emiliano Ricardo

2006 "Informe. Análisis de huellas de manufactura de la lapidaria de Teopancazco y Xalla, Teotihuacán”. México, Archivo del Proyecto Teotihuacán: Élite y 
Gobierno, Universidad Nacional Autónoma de México, Instituto de Investigaciones Antropológicas.

2009a "Análisis tecnológico de los objetos de piedra verde del Templo Mayor de Tenochtitlan", Memorias del 53 Congreso Internacional de Americanistas, México: Instituto Nacional de Antropología e Historia-Universidad Iberoamericana (versión digital en CD).

2009b "La producción especializada de objetos de concha en Xochicalco", tesis de Maestría en Antropología. México: Universidad Nacional Autónoma de México.

Melgar Tísoc, Emiliano Ricardo y Reyna Beatriz Solís Ciriaco

2011 "Caracterización de huellas de manufactura en objetos lapidarios de obsidiana del Templo Mayor de Tenochtitlan”, Arqueología, 42: 118-134. México: Raíces.

Melgar Tísoc, Emiliano, Reyna Solís Ciriaco y Ernesto González Licón

2010 "Producción y prestigio en concha y lapidaria de Monte Albán", Producción de bienes de prestigio ornamentales y votivos de la América antigua, Emiliano Melgar Tísoc, Reyna Solís Ciriaco y Ernesto González Licón (eds.). Miami: Syllaba Press, pp. 6-21.

Mirambell, Lorena

1968 Técnicas lapidarias prehispánicas. México: Instituto Nacional de Antropología e Historia.

Moholy-Nagy, Hattula

1997 "Middens, Construction Fill, and Offerings: Evidence for the Organization of Classic Period Craft Production at Tikal, Guatemala", Journal of Field Archaeology, 24: 293-313. USA: W.S. Maney \& Son.

Nelson, Zachary, Barry Scheetz, Guillermo Mata Amado y Antonio Prado

2009 "Composite Mirrors of the Ancient Maya: Ostentatious Production and Precolumbian Fraud", The PARI Journal. A quarterly publication of the Pre-Columbian Art Research Institute, IX (4): 1-16. San Francisco, CA: Pre-Columbian Art Research Institute.

Pastrana, Alejandro

1998 La exploración de la obsidiana en la Sierra de las Navajas. México: Instituto Nacional de Antropología e Historia.

Pereira, Gregory

2008 "La materia de las visiones. Consideraciones acerca de los espejos de pirita prehispánicos”, Diario de Campo, mayo/junio (48): 123-136.

Pfaffenberger, Bryan.

1988 "Fetishised Objects and Humanised Nature: Towards an Anthropology of Technology”, Man, 23: 236-252. Great Britain: Royal Anthropological Institute of Great Britain and Ireland. 
Pires-Ferreira, Jane W.

1975 Formative Mesoamerican Exchange Networks with Special Reference to the Valley of Oaxaca. Prehistory and Human Ecology of the Valley of Oaxaca, Ann Arbor: University of Michigan, vol. 3.

Pires-Ferreira, Jane W. y Billy Joe Evans

1978 "Mössbauer Spectral Analysis of Olmec Iron Ore Mirrors: New Evidence of Formative Period Exchange Networks", Cultural Continuity in Mesoamerica, David L. Browman (ed.). The Hague: Mouton, pp. 101-154.

Rochette, Erick T.

2009 "Jade in Full: Prehispanic Domestic Production of Wealth Goods in the Middle Motagua Valley, Guatemala”, Archaeological Papers of the American Anthropological Association, 19 (1): 205-224, Kenneth G. Hirth (ed.). Nueva Jersey: American Anthropological Association.

Sahagún, fray Bernardino de

1956 Historia general de las cosas de la Nueva España. México: Porrúa.

Salinas, Flores.

1995 Tecnología y diseño en el México prehispánico. México, Universidad Nacional Autónoma de México, Facultad de Arquitectura, Centro de Investigaciones de Diseño Industrial.

Semenov, S. A.

1957 Prehistoric Technology, an Experimental Study of the oldest Tools and Artefacts from traces of Manufacture and Wear. Londres: Cory, Adams \& MacKay.

Schiffer, Michael B.

1992 Technological Perspectives on Behavioral Change. Tucson: University of Arizona Press.

Shimada, Izumi

1994 "Introducción”, Tecnología y Organización de la Producción de Cerámica Prehispánica en los Andes. Lima: Pontificia Universidad Católica del Perú, pp. 13-31.

Smith, A. Ledyard y Alfred V. Kidder

1951 Excavations at Nebaj, Guatemala. Washington, D. C.: Carnegie Institution of Washington (Publication, 594).

Sugiyama, Saburo

1992 "Rulership, Warfare, and Human Sacrifice at the Ciudadela: An Iconography Study of the Feathered Serpent Representation", Art, Ideology, and the City of Teotihuacan, Janet Berlo (ed.). Washington D.C.: Dumbarton Oaks Research Library and Collection, pp. 205-230. 
Taube, Karl A.

1992 "The Iconography of Mirrors at Teotihuacan", Art, Ideology, and the City of Teotihuacan, Janet Berlo (ed.). Washington D.C.: Dumbarton Oaks Research Library and Collection, pp. 169-204.

Tringham, Ruth

1978 "Experimentation, Ethnoarchaeology, and the Leapfrog in Archaeological Methodology", Explorations in ethnoarchaeology, Richard A. Gould (ed.). Albuquerque: University of New Mexico Press, pp. 169-199.

Turner, Margaret $\mathrm{H}$.

1992 "Style in Lapidary Technology: Identifying the Teotihuacan Lapidary Industry", Art, Ideology, and the City of Teotihuacan, Janet Berlo (ed.). Washington D.C.: Dumbarton Oaks Research Library and Collection, pp. 89-112.

Velázquez Castro, Adrián

1999b "Arqueología experimental en materiales conquiológicos. Informe final". México: Museo del Templo Mayor, mecanoescrito.

2007 La producción especializada de los objetos de concha del Templo Mayor de Tenochtitlan. México: Instituto Nacional de Antropología e Historia (Colección Científica, 519)

Weigand, Phil

1995 "Minería prehispánica en las regiones noroccidentales de Mesoamérica con énfasis en la Turquesa”, Arqueología del Occidente y Norte de México, Eduardo Williams y Phil Weighand (eds.), Zamora: El Colegio de Michoacán, pp. 115138.

1997 “La Turquesa”. Arqueología Mexicana, V (27): 26-36. México: Raíces.

Widmer, Randolph J.

2009 "Elite Household Multicrafting Specialization at 9N8, Patio H, Copan", Archaeological Papers of the American Anthropological Association, 19 (1): 174204, Kenneth G. Hirth (ed.). Nueva Jersey: American Anthropological Association.

Wobst, H. Martin.

1977 "Stylistic Behavior and Information Exchange", For the Director. Research Essay in Honor of James B. Griffen, Charles E. Cleland (ed.). Ann Arbor: University of Michigan, pp. 317-342.

Woodward, Arthur

1941 “Hohokam Mosaic Mirrors”, Los Angeles County Museum Quarterly, 1 (4): 6-11. Los Ángeles, CA: Museum Association.

Zamora, Fabián Marcelo

2002a "La industria de la pirita en el sitio de Aguateca durante el periodo Clásico Tardío", tesis de Licenciatura en Arqueología. Guatemala: Universidad del Valle de Guatemala. 
2002b "La industria de la pirita en el sitio Clásico Tardío de Aguateca”, XV Simposio de Investigaciones Arqueológicas en Guatemala, 2001, J. P. Laporte, H. Escobedo y B. Arroyo (eds.). Guatemala: Museo Nacional de Arqueología y Etnología, pp. 695-708. 